\title{
The Association of Lipoprotein-a Levels, Neutrophil Lymphocyte Ratio and Hypertension with the Clinical Severity Scale Measured by NIHSS Scale in Patients with Acute Thrombotic Stroke
}

\author{
Muhammad Hamdan, Moh. Hasan Machfoed, Paulus Sugianto, A. Firdaus Sani, Moh Saiful Ardhi, Fadil \\ Department of Neurology, Faculty of Medicine, \\ Dr. Soetomo Teaching Hospital Surabaya-Universitas Airlangga 60285, Indonesia
}

\begin{abstract}
Background: The correlation between levels of Lipoprotein-a, Neutrophil Lymphocyte Ratio and hypertension with clinical severity scale remains a controversial issue.

Objective: To determine the correlation of levels of Lipoprotein-a, Neutrophil Lymphocyte Ratio and hypertension with the severity scale measured by NIHSS scale in patients with acute thrombotic stroke.

Method: Lipoprotein-a levels, Neutrophil Lymphocyte ratio, blood pressure in patients with acute thrombotic stroke were measured and clinical severity scale was assessed by NIHSS scale. The levels of lipoprotein-a were grouped into normal and high levels of lipoprotein-a, Lymphocyte Neutrophil Ratios were grouped into low and high and hypertension were grouped into stage 1 and 2 . The data was analyzed using logistic regression.

Results: There were 40 patients consisting of 29 (72.50\%) male patients and 11 (27.50\%) female patients. The demographic data included gender, age, LDL level, random blood sugar level, diabetes mellitus status and smoking status which were all homogeneous in both groups of lipoprotein-a, Neutrophil Lymphocyte ratio and hypertension stage. In the logistic regression analysis, the lipoprotein-a and neutrophil lymphocyte ratio were correlated with clinical severity scale $(\mathrm{p}=0.018$, RO $0.122(\mathrm{CI} 95 \% 0.022-0.696)$ vs $\mathrm{p}=0.041$, RO 0.068 (95\% CI 0.005-0.895) while hypertension stage was not correlated with clinical severity scale (p $=0.97$, RO $1.02(95 \%$ CI 0.28-3.80).
\end{abstract}

Conclusion: The levels of lipoprotein-a and lymphocyte neutrophil ratio were related to the clinical severity measured by NIHSS scale but not with hypertension.

Keywords: Lipoprotein-a, Lymphocyte Neutrophil Ratio, Hypertension, NIHSS, Stroke.

\section{Introduction}

Stroke is the fourth leading cause of death in the

\section{Corresponding Author:}

Muhammad Hamdan

Department of Neurology, Faculty of Medicine, Dr.

Soetomo Teaching Hospital Surabaya-Universitas

Airlangga 60285, Indonesia
United States. In addition, it is the main cause of disability in Indonesia, including in Dr. Soetomo General Hospital Surabaya with a fairly high mortality rate of $28.76 \%^{1-3}$. Various risk factors of stroke have been known in which hypertension and elevated levels of Lipoprotein-a is one of the modifiable risk factors ${ }^{4}$. Increased levels of Lipoprotein-a (Lp-a) is one of the potential stroke risk factors that can be modified. Lipoprotein-a is a complex particle composed of LDL molecules as nuclei with apolipoprotein-b and apolipoprotein-a bound by a 
disulfide bridge ${ }^{5}$. High levels of lipoprotein-a result in the process of atherotrombosis by various mechanisms such as increasing ICAM expression, inhibiting TGF-b, increasing the production of IL-6 and IL- $8^{6}$. In patients with hypertension and stroke, Lipoprotein-a levels have increased where the normal limit is ${ }^{1} 30 \mathrm{mg} / \mathrm{dl}$. These levels are associated with the occurrence of carotid atherosclerotic. The greater the level of Lipoprotein-a, the greater the degree of atherosclerosis in the carotid artery in patients with ischemic stroke ${ }^{7}$. Levels of lipoprotein-a are not related to the severity scale and clinical outcomes in patients with acute ischemic stroke $^{8}$. The levels of Lipoprotein-a, IL-6, albumin and ferritin are related to the degree of severity and clinical outcomes in patients with ischemic stroke.

Inflammation plays an important role in the pathophysiology of acute ischemic stroke. This inflammatory process results in endothelial damage and blood brain barrier, accumulation of inflammatory mediators, large leukocyte and platelet infiltration ${ }^{7}$.On the other hand, lymphocytes, primarily $\mathrm{T}$ lymphocytes, infiltrate around the ischemic zone from day 3 and increase on day 3 to day $7^{9}$. The role of lymphocytes in stroke is to decrease the proliferation and cytolytic activity of IL-2 and mitogen. The severity of neurological deficits in early stroke is associated with low $\mathrm{T}$ lymphocyte levels. This suggests a suppression of immune responses in patients with ischemic stroke $\left.{ }^{10}\right)$. Clinical studies show that neutrophil accumulation in cerebral infarction is associated with the severity of brain tissue damage and poor neurologic outcome after an ischemic stroke 9 . High neutrophil levels are associated with an increased NIHSS scale at the onset of stroke ${ }^{11}$.

The Neutrophil Lymphocyte ratio (NLR) is a potential new biomarker for the risk of cardiovascular disease. NLR is an independent factor of the severity of coronary heart disease and decreased life expectancy in patients with coronary heart disease ${ }^{12}$ ) Increased NLR values are a predictor factor of 30-day mortality in patients with acute coronary syndrome ${ }^{13}$. NLR has similar sensitivity and specificity to hs-CRP (sensitivity vs specificity of hs-CRP: $84 \%$ vs $83 \%)^{12}$.

In acute ischemic stroke, NLR has not been widely studied and each study has different limitation values. NLR value ${ }^{1} 3.17$ is associated with a risk of stroke in non valvular atrial fibrillation patients ${ }^{14}$ ). Another study mentioned that the NLR value of 4.5 is associated with acute ischemic stroke ${ }^{15}$. For stroke output, the NLR value of ${ }^{1} 5.9$ is associated with poor clinical outcomes and death within 90 days ${ }^{16}$. Another study showed that the NLR value of $\geq 5$ is associated with clinical severity and short-term mortality within 60 days of non-lunar stroke patients compared to stroke lakunerv ${ }^{13}$.

Various observational data indicate that an increase in blood pressure during acute stroke is associated with a poor prognosis ${ }^{17}$. In lacuner stroke patients, an increase in systolic blood pressure between 140-220 $\mathrm{mmHg}$ and diastolic blood pressure between 70-110 has a better clinical severity scale compared with other stroke types $^{18}$.

The aim of this study was to prove the association between lipoprotein-a, NLR and hypertension with clinical severity as measured by nihss scale in acute thrombotic stroke.

\section{Method}

This study is a cross-sectional study with consecutive sampling with primary data of patients with acute thrombotic stroke of first attack and onset $<8$ days. The subjects were collected from Dr. Soetomo General Hospital Surabaya, Indonesia without sepsis, haematological disorders, malignancy, corticosteroid and immunosuppressant, elevated liver enzyme levels, renal failure, cardiac arrhythmia, congestive heart failure and acute myocardial infarction. The total subjects in this study were 40 patients.

The levels of Lipoprotein-a, Neutrophil Lymphocyte Ratio and hypertension were examined at $<8$ days of onset and observed its associations among subjects with normal $(£ 18 \mathrm{mg} / \mathrm{dl})$ and high Lp-a $(>18 \mathrm{mg} / \mathrm{dl})$, low $(£ 4,5)$ and high NLR $(>4.5)$ and hypertension stage 1 $\left({ }^{1} 140-159 / 90-99\right)$ and Stage $2\left({ }^{1} 160 / 100\right)$ with clinical severity measured using NIHSS scale classified as mild $(0-£ 4)$, moderate $(5-15)$ and severe $(>15)$ with logistic regression analysis using SPSS 21.0 program (SPSS, Inc., Chicago, IL).

\section{Results}

During the period of 3 (three) months, there were 40 research subjects who fulfilled inclusion criteria and no subjects who were excluded. It consisted of 29 males $(72.5 \%)$ and 11 females $(27.5 \%)$. 
Table 1. The Bivariate Analysis of Research Variables with Clinical Severity Scales

\begin{tabular}{|c|c|c|c|c|c|c|}
\hline \multirow{2}{*}{ Variables } & \multicolumn{2}{|c|}{ Clinical severity } & \multirow{2}{*}{$\begin{array}{l}\text { Total } \\
\text { n (\%) } \\
\end{array}$} & \multirow{2}{*}{$\mathbf{p}$} & \multirow{2}{*}{ RO } & \multirow{2}{*}{ IK 95\% } \\
\hline & Mild (n (\%)) & Moderate (n (\%)) & & & & \\
\hline \multicolumn{7}{|l|}{ Lp-a level } \\
\hline Normal & $9(64.29)$ & $5(35.71)$ & $14(100)$ & 0.04 & 4.05 & $1.03-16.01$ \\
\hline High & $8(30.77)$ & $18(68.23)$ & $26(100)$ & & & \\
\hline \multicolumn{7}{|l|}{ NLR } \\
\hline Low & $16(48.49)$ & $17(51.51)$ & $33(100)$ & 0.21 & 5.63 & $0.61-52.22$ \\
\hline High & $1(14.29)$ & $6(85.71)$ & $7(100)$ & & & \\
\hline \multicolumn{7}{|l|}{ Hypertension } \\
\hline Stage 1 & $6(42.86)$ & $8(57.14)$ & $14(100)$ & 0.97 & 1.02 & $0.28-3.08$ \\
\hline Stage 2 & $11(42.31)$ & $15(57.69)$ & $26(100)$ & & & \\
\hline \multicolumn{7}{|c|}{ Random glucose level } \\
\hline Normal & $16(44.44)$ & $20(55.56)$ & $36(100)$ & 0.62 & 2.40 & $0.23-25.34$ \\
\hline High & $1(25)$ & $3(75)$ & $4(100)$ & & & \\
\hline \multicolumn{7}{|l|}{ Smoking status } \\
\hline Non smoker & $9(42.86)$ & $12(57.14)$ & $21(100)$ & 0.96 & 1.03 & $0.29-3.62$ \\
\hline Smoker & $8(42.11)$ & $11(57.89)$ & $19(100)$ & & & \\
\hline \multicolumn{7}{|l|}{ Age } \\
\hline$£ 55$ years old & $6(46.15)$ & $7(53.85)$ & $13(100)$ & 0.75 & 1.25 & $0.33-4.73$ \\
\hline$>55$ years old & $11(40.74)$ & $16(59.26)$ & $27(100)$ & & & \\
\hline \multicolumn{7}{|l|}{ Gender } \\
\hline Male & $12(41.38)$ & $17(58.62)$ & $29(100)$ & 1.00 & 0.85 & $0.21-3.43$ \\
\hline Female & $5(45.46)$ & $6(54.54)$ & $11(100)$ & & & \\
\hline
\end{tabular}

Bivariate analysis with Chi-Square between Lp-a levels, Neutrophil Lymphocyte Ratio and Hypertension and bivariate analysis of confounding factors (glucose levels, smoking status, age and gender) with clinical severity measured by NIHSS scales presented in table 2 . The factors correlated to or affected the clinical severity scale were Lp-a and NLR and can be incorporated into logistic regression analysis $(\mathrm{p}<0.25)$ whereas hypertension, hyperglycemia, smoking, age and gender did not affect the clinical severity and can not be incorporated into logistic regression analysis $(\mathrm{p}>0.25)$.

Table 2. The Logistic Regression Analysis of the Association between Lp-a Level and NLR with the Clinical Severity Scales

\begin{tabular}{|l|l|c|c|c|}
\hline & Variable & Coefficient & p & RO (IK 95\%) \\
\hline Step 1 & Lp-a level & 2.100 & 0.018 & $0.122(0.022-0.696)$ \\
\hline & NLR value & 2.692 & 0.041 & $0.068(0.005-0.895)$ \\
\hline & Constant & 3.341 & 0.014 & 28.235 \\
\hline
\end{tabular}

NIHSS $=3.341+(2.100 \times$ Lp-a $)+(2.692 \times$ NLR $)$

Note $=$ Ifnormal $\mathrm{Lp}-\mathrm{a}=0$, high $\mathrm{Lp}-\mathrm{a}=1$, Low NLR $=0$, High NLR $=1$

Table 2 shows a multivariate analysis of logistic regression to determine the association or effect of Lp-a levels and NLR with the clinical severity in acute thrombotic stroke patients. Lp-a levels and NLR correlated or affected the degree of clinical severity and obtained a good calibration regression equation (p>0.05) (Hosmer and Lame show Test, $p=0.94)$ and low discrimination value (Area Under Curve (AUC) of $26.2 \%)$. 


\section{Discussion}

We found an association between the levels of Lipoprotein-a and Neutrophil Lymphocyte Ratio with the clinical severity measured by NIHSS scale. However, there was no correlation between hypertension and the clinical severity measured by NIHSS scale in acute thrombotic stroke patients.

This study revealed that males had higher level of Lp-a than females. Previous research has shown that Lp-a levels in male patients with thrombotic stroke are higher than female patients ${ }^{19}$. Lp-a levels in normal individuals were $16.4 \mathrm{mg} / \mathrm{dl}$ and Lp-a levels in females were higher than in males (20.9 $\mathrm{mg}$ vs $11.3 \mathrm{mg} / \mathrm{dl}$ ). Increased levels of Lp-a increase the risk of cerebro and cardiovascular disease ${ }^{20}$. Lp-a levels are not affected by gender $^{21}$. Neutrophil Lymphocytes Ratio of this study was not influenced by gender. This is supported by previous research which stated that the value of NLR did not obtain significant differences $b$ between male and female subjects ${ }^{13}$. Hypertension stage 1 and 2 occurred more in males than females, but there was no significant difference $(p=0.47)$. Researches in China and Bosnia reported that female patients with acute thrombotic stroke more suffer from hypertension than male patients. However, $>80$ year-old male are more likely to suffer from hypertension than females ${ }^{22}$.

LDL levels affect Lp-a levels ${ }^{21-22}$. In this study, the LDL levels were evenly distributed (homogeneous) in both normal Lp-a and high Lp-a groups and there was no significant difference between the two groups. Likewise, LDL levels of NLR and hypertension did not cause any bias.

The random glucose levels in the normal and high Lp-a group, low and high NLR scores and stage 1 and 2 hypertension were not statistically significant. It implies that random glucose levels are homogeneously distributed in both groups. Hyperglycemia in patients with diabetes mellitus can primarily cause the formation of thrombus in the arteries, which can lead to abnormalities of cerebro and cardiovascular ${ }^{23}$. Hyperglycemia occurs in $30-40 \%$ of stroke patients without previous diabetes mellitus and hyperglycemia history during hospitalization due to the poor outcome ${ }^{24}$.

The characteristics of subjects based not on diabetes mellitus and on diabetes mellitus at both normal and high Lp-a levels, NLR values and hypertension stages were homogeneously distributed in both groups marked with $\mathrm{p}>0.05$. Lp-a levels between people with diabetes mellitus and without diabetes mellitus showed no significant difference ${ }^{25}$ ohirA. Patients with acute ischemic stroke with diabetes mellitus are often accompanied by other risk factors of hypertension and there is a relationship between increased blood sugar levels in diabetes mellitus with hypertension ${ }^{26}$.

Smoking is a risk factor of stroke. In this study, smoking status in normal and high Lp-groups, low and high NLR groups and hypertension stages were homogeneously distributed. Smoking and hypertension lead to intracranial stenosis ${ }^{27}$ Smoking is not associated with Lp-a levels erqou ${ }^{28}$.

Multivariate analysis of logistic regression was performed to determine the relationship between Lp-a and NLR values with the clinical severity, whereas hypertension stage could not be included in multivariate analysis. In addition, an equation can be used to predict the clinical severity of acute thrombotic stroke. In the multivariate analysis, it was found that Lp-a and NLR values were correlated with the clinical severity with values of $p<0.05$ and the obtained equation could predict the degree of clinical severity by entering Lp-a and NLR values due to the good calibration and discrimination. However, it was considered statistically very weak value of AUC. In this study, it was revealed that patients with acute thrombotic stroke had high Lp-a levels and NLR values for more severe severity of $26.2 \%$. The normal Lp-a and low NLR levels were protective for more severe clinical severity.

Normal Lp-a levels are physiologically functioning in wound healing, angiogenesis and hemostasis. Lipoprotein-a is a source of cholesterol used for repair of tissue regeneration. In the angiogenesis process, lipoprotein-a is important for sprouting new blood vessels and in the process of hemostasis, competitively inhibiting plasminogen binding to fibrinogen and fibrin and inhibiting fibrinogen activation depending on fibrin through tissue plasminogen activator. In addition, lipoprotein-a binds platelet activating acetylhidrolase factor which is the strongest factor of platelet aggregation and hydrolyzes phospholipids during the lipid oxidation process and inhibits thromboxan secretion..

\section{Conclusion}

It can be concluded that there is an association between the levels of Lipoprotein-a and Neutrophil Lymphocyte Ratio with the clinical severity measured 
by NIHSS scale. However, there is no correlation between hypertension and the clinical severity measured by NIHSS scale in acute thrombotic stroke patients.

Ethical Clearance: This study received an ethical test from Dr. Soetomo General Hospital and faculty of medicine Universitas Airlangga.

Source of Funding: This research was carried out through individual funding.

Conflict of Interest: There was no conflict of Interest from this study.

\section{References}

1. Go AS, Mozaffarian D, Roger VL, Benjamin EJ, Berry JD, Blaha MJ et al. Heart disease and stroke statistics--2014 update: a report from the American Heart Association. Circulation. 2014; 129(3):e28292.

2. Kementrian Kesehatan Republik Indonesia. Menkes Lakukan Soft Opening Rumah Sakit Pusat Otak Nasional. 2013.

3. MS I. Acute Ischemic Stroke In Surabaya. In: Islam MS SA, Subadi I et al, (Eds) In: Postgraduate Course on Neurology and Rehabilitation Medicine, editor. 2013.

4. Goldstein LB, Bushnell, C.D., Adams, R.J. et al. G. Guidelines for the primary prevention of stroke: a guideline for healthcare professionals from the American Heart Association/American Stroke Association. Stroke [Internet]. 2011;42(2):517-84.

5. Plow JH, Huang M. Lipoprotein (a) Metabolism : Potential site for Therapeutic Targets. 2013;62:47991. Metab Clin Exp. 2013;62:479-91.

6. Siekmeier R, Scharnagl, H., Kostner, G.M. et al. Lipoprotein (a)- Strukture, Epidemiology, Function and Diagnostic of Cardiovascular Marker. Open Clin Chem J. 2008; 1:79-91.

7. Nasr N, Ruidavets, J.B., Farghali, A. et al. Lipoprotein (a) and Carotid Atherosclerosis in Young Patients With Stroke. Stroke [Internet]. 2011;42:3616-8.

8. Kooten F, Krimpen, J., Dippel, D.W.J. et al. Lipoprotein (a) in Patients With Acute Cerebral Ischemia. Stroke [Internet]. 1996;27:1231-5.

9. Jin R, Yang, G., Li G. Inflammatory Mechanisms in Ischemic Stroke: Role of Inflammatory Cells. J Leukoc Biol. 2010;87:779-89.
10. Yilmaz G, Granger DN. Leukocyte Recruitment and Ischemic Brain Injury. Neuromolecular Med. 2010; 12:193-204.

11. Kim J, Song, T.J., Park, J.H. et al. Different Prognostic Value of White Blood Cell subtypes in Patients with Acute Cerebral Infarction. Atherosclerosis. 2012;222:464-7.

12. Akpek M, Kaya, M.G., Lam, Y.Y. et al. Relation of Neutrophil/Lymphocyte Ratio to Coronary Flow to in-Hospital Major Adverse Cardiac Events in Patients with ST-Elevated Myocardial Infarction Undergoing Primary Coronary Intervention. Am J Cardiol. 2012; 110:621-7.

13. Tokgoz S, Kayrak, M., Akpinar, Z. et al. Neutrophil Lymphocyte Ratio as a Predictor of Stroke. J stroke Cerebrovasc Dis. 2013;20:1-6.

14. Ertas G, Sonmez, O., Turfan, M. et al. Neutrophil/Lymphocyte Ratio is Associated with Thromboembolic Stroke in Patients with Non-valvular Atrial Fibrillation. J Neurol Sci. 2013;324:49-52.

15. Gokhan S, Ozhasenekler, A., Durgun, H.M. et al. Neutrophil Lymphocyte Ratio in Stroke Subtype and Transient Ischemic Attack. Eur Rev Med Pharmcological Sci. 2013; 17:653-7.

16. Brooks SD, Spears, C., Cummings, C. et al. Admission Neutrophil-Lymphocyte Ratio Predict 90 Day Outcome after Endovascular Stroke Therapy. J Neurointerv Surg. 2013; 10:11-36.

17. Wityk RJ. Blood Pressure Augmentation in Acute Ischemic Stroke. J Neurol Sci. 2007;261:63-73.

18. Semplecini A, Maresca, A., Boscolo, G. et al. Hypertension in Acute A Compensatoy Mechanism or an Additional Damaging Factor? Arch Intern Med. 2003; 163:211-6.

19. Dhamija RK, Gaba, P., Arora, S. et al. Homocysteine and Lipoprotein (a) Correlation in Ischemic Stroke Patients. J Neurol Sci. 2009;281:64-8.

20. Oliveira SHV, Miranda, M.R., Morais, C. A.S. et al. Serum Lipoprotein (a) Level in Healthy Subject Indicate a Lurking Cerebro-and Cardio-Vascular Risk in the Younger Populations. Brain Res Bull. 2013;97:48-52.

21. Misirli H, Somay, G., Ozbal, N. et al. Relation of Lipid and Lipoprotein (a) to Ischaemic Stroke. J Clin Neurosci. 2002;9:127-32.

22. Salihovic D, Smajlovic, D., Sinanovic, O. et al. Sex 
Differences in Patients with Acute Ischemic Stroke in Tuzla Region, Bosnia and Herzegovina. Bosn J Basic Med Sci. 2010; 10:116-21.

23. Hansen HR, Wolfs, J.L., Bruggemann, L. et al. Hyperglycemia accelerates arterial thrombus formation and attenuates the antithrombotic response to endotoxin in mice. Blood Coagul fibrinolysis an Int J Haemost Thromb. 2007; 18:627-36.

24. Luitze MA, Biessels, G.J., Rutten, G. et al. Diabetes, Hyperglicemia and Acute Ischemic Stroke. Lancet Neurol. 2012; 11:261-71.

25. Ohira T, Schreiner, P.J., Morrisett, J.D. et al. Lipoprotein (a) and Incident Ischemic Stroke:
The Atherosclerosis Risk in Communities (ARIC) Study. Stroke [Internet]. 2006;37:1407-12.

26. AH A-K. The incidence of stress hyperglycemia in acute ischemic stroke patients (in Al-Yarmouk teaching hospital). Mustansiriya Med J. 2012; 11111111:46-51.

27. Kim DE, Lee, K.B., Jang, I.M. et al. Associations of cigarette smoking with intracranial atherosclerosis in the patients with acute ischemic stroke. Clin Neurol Neurosurg. 2012; 114:1243-7.

28. Erqou S, Kaptoge, S., Perry, P.L. et al. Lipoprotein (a) Concentration and the Risk of Coronary Heart Disease, Stroke and Nonvascular Mortality. JAMA. 2009;302:412-23. 